\title{
Moluscos planctónicos entre el fiordo Reloncaví y el golfo Corcovado, sur de Chile: ocurrencia, distribución y abundancia en invierno
}

Planktonic mollusks between the Reloncaví Fjord and the Corcovado Gulf, southern Chile: winter occurrence, distribution and abundance

\section{Bernardita Campos ${ }^{1}$ y Mauricio F. Landaeta ${ }^{1}$}

'Escuela de Biología Marina, Facultad de Ciencias del Mar y de Recursos Naturales, Universidad de Valparaíso, casilla 5080, Reñaca, Viña del Mar, Chile. bernardita.campos@uv.cl

\begin{abstract}
Despite their important ecological functions, knowledge of planktonic mollusks in southern fjords of Chile is limited. Hence, the goals of this research were to determine the composition of the main groups of planktonic mollusks, their geographical distribution and abundance, between the Reloncavi Fjord and the Corcovado Gulf. Plankton samples were collected at 16 oceanographic stations during the CIMAR-19 Fiordos cruise, in July 2013. Oblique hauls were made in two water depth layers according to the pycnocline depth with a WP2 net (180 $\mu \mathrm{m}$ mesh size). The organisms were removed manually from each sample, counted and assigned to the lowest possible taxonomic level. Mollusks were obtained in all stations: Bivalvia (39.68\%), Gastropoda $(60.31 \%)$ and Cephalopoda (0.01\%). The total abundance of mollusks varied between 18.46 and $124.42 \mathrm{ind}^{\mathrm{m}}$. Among bivalves highlighted the abundance of Pholadidae, Teredinidae and Xylophagidae larvae. Among gastropods highlighted the abundance of Thecosomata and Nudibranchia. Among cephalopods, only Octopoda paralarvae were captured. The abundance of bivalve boring species and their larvae have an important role in the flow of allochthonous organic matter across the fjords. The water temperature and salinity may be the variables that mostly modulate the distribution of planktonic bivalves and gastropods in the Chilean fjords.
\end{abstract}

Key words: Meroplankton, holoplankton, larval ecology, Southeast Pacific, Chilean fjords

\begin{abstract}
Resumen.- A pesar de sus importantes funciones ecológicas, el conocimiento de los estados planctónicos de moluscos en fiordos australes de Chile es limitado. De aquí que los objetivos de esta investigación fueron determinar la composición de los principales grupos de moluscos planctónicos, su distribución geográfica y abundancia, entre el fiordo Reloncaví y el golfo Corcovado. Las muestras de plancton se recolectaron en 16 estaciones oceanográficas, durante el Crucero CIMAR 19-Fiordos, en julio 2013. Se efectuaron lances oblicuos en 2 estratos, de acuerdo con la profundidad de la picnoclina con una red WP2 (180 $\mu \mathrm{m}$ abertura de malla). Los organismos fueron separados manualmente de cada muestra, contados y asignados al menor nivel taxonómico posible. Se obtuvieron moluscos en todas las estaciones: Bivalvia (39,68\%), Gastropoda (60,31\%) y Cephalopoda (0,01\%). La abundancia total varió entre 18,46 y 124,42 ind. $\mathrm{m}^{-3}$. Destacó la abundancia de larvas de bivalvos Pholadidae, Teredinidae y Xylophagidae. De gasterópodos destacó la abundancia de Thecosomata y Nudibranchia. De cefalópodos, solo se capturaron paralarvas de Octopoda. La alta abundancia relativa de larvas de bivalvos perforadores de madera sería un indicador de la presencia de materia orgánica de origen forestal en el fondo de los fiordos y tendrían un rol importante en el flujo de materia orgánica alóctona que llega a los fiordos. La temperatura y salinidad del agua serían las variables que mayormente modularían la distribución de bivalvos y gasterópodos planctónicos en los fiordos chilenos.
\end{abstract}

Palabras clave: Meroplancton, holoplancton, ecología larval, Pacífico sureste, fiordos chilenos

\section{INTRODUCCIÓN}

Los moluscos planctónicos son importantes en la dinámica trófica acuática, en la dispersión geográfica de las poblaciones adultas, en el manejo pesquero y en la conservación de la biodiversidad (Botsford et al. 2001, Shanks 2001, Young 2002, Trakhtitenbrot et al. 2005). En el plancton, los moluscos están representados mayoritariamente por estados larvales (meroplancton) de especies que en sus estados juvenil y adulto habitan el bentos como ocurre con la mayoría de las especies de bivalvos y de gasterópodos (Shanks 2001, Zardus \& Martel 2002, Buckland-Nicks et al. 2002) y además, por la totalidad de los estados del desarrollo de las especies de moluscos que permanecen toda su vida en la columna de agua (holoplancton), como los gasterópodos thecosomados (Suárez-Morales et al. 2009) y las paralarvas de cefalópodos nectónicos (Vecchione \& Shea 2002) 
Para comprender la dinámica de las poblaciones bentónicas de moluscos (así como de otros invertebrados) y su conectividad, es fundamental el conocimiento de la distribución de sus estados pelágicos, su potencial de dispersión, las escalas en que esta se presenta y los factores oceanográficos que la modulan (Thorson 1966, McEdward 1995, Fernández et al. 2009, Navarrete et al. 2015). La distribución de los estados pelágicos es el resultado de la interacción de procesos pasivos (advección) y activos (natación) que ocurren a distintas escalas espacio-temporales. Por una parte, los organismos se dispersarían pasivamente dependiendo de las corrientes y, por otra, regularían activamente su posición vertical en respuesta a discontinuidades fisicoquímicas de la columna de agua. La posición vertical de los organismos en la columna de agua es de gran trascendencia para su retención o exportación hacia áreas adecuadas ya que el ambiente hidrodinámico al cual están expuestas, es un componente esencial tanto para un asentamiento exitoso al bentos en el caso de las larvas (Butman 1987) como para la supervivencia de todos los estados del desarrollo, como ocurre en los moluscos holoplanctónicos (Bernard 2006). Por lo tanto, las observaciones sobre la distribución espacial y temporal de los organismos planctónicos, especialmente de las larvas, son importantes para realizar estimaciones de dispersión y establecer aún, de manera indirecta, con base en su presencia o ausencia, los períodos reproductivos de las especies presentes en un área geográfica determinada.

Fiordos y canales del sur de Chile presentan una alta diversidad biológica asociada principalmente con las características físico-químicas de las masas de agua. Las cuencas conectadas al mar son más amplias y profundas y tienen mayor productividad y biomasa que las cuencas interiores, de menor profundidad y de dimensiones relativamente menores (Pavés et al. 2014). La comunidad macrozooplanctónica de fiordos y canales del sur del país ha sido bastante estudiada gracias a los proyectos CIMAR (Palma 2006), sin embargo, han recibido mucho menor atención el meso y microzooplancton, fracciones con representantes de numerosas especies de moluscos. Entre los estudios acerca de moluscos planctónicos (larvas, paralarvas y holoplancton) en los fiordos chilenos, se cuentan los aportes de Solís et al. (1976), Uribe et al. (1982), Antezana et al. (1996, 1999), Vega et al. (2000), Molinet et al. (2005, 2006, 2008), Campos \& Díaz (2007), Molinet \& Moreno (2009), Balbontín et al. (2009), Marilao (2009), Avendaño et al. (2011), Barría et al. (2012), Carrasco et al. (2012), Ojeda et al. (2014) ${ }^{1}$, Meerhoff et al. (2014) y PardoGandarillas et al. (2016).
La ocurrencia de adultos bentónicos de moluscos, en la zona de fiordos y canales del sur de Chile, ha sido bien estudiada en las últimas décadas (Schörld 1997, Osorio 1999, Valdovinos 1999, Reid \& Osorio 2000, Osorio et al. 2002, 2005, 2006; Osorio \& Reid 2004, Valdovinos \& Rüth 2005, Schwabe $e t$ al. 2006, Osorio \& Ramajo 2007, Cárdenas et al. 2008, Häussermann \& Försterra 2009, Vega 2009, Ramajo \& Osorio 2012, Güller \& Zelaya 2015), registrándose varias especies que constituyen recursos pesqueros. Para la conservación y el manejo sostenible de áreas productivas como los canales y fiordos y de las especies de moluscos que las habitan, es indispensable disponer de información acerca de la ocurrencia, distribución y abundancia de sus estados planctónicos, para aportar más antecedentes para el manejo y conservación de este grupo zoológico en la zona. Los objetivos de esta investigación fueron determinar la composición, distribución geográfica y abundancia, de los grupos principales de moluscos planctónicos entre el estero de Reloncaví y el golfo Corcovado, en los fiordos del sur de Chile.

\section{Materiales Y MÉTOdos}

Los moluscos fueron obtenidos de muestras de plancton recolectadas durante el desarrollo del Crucero CIMAR 19Fiordos, por el buque de investigación AGS 'Cabo de Hornos', de la Armada de Chile, en 16 estaciones de muestreo (Tabla 1), entre el fiordo de Reloncaví (4140'44,4' S-

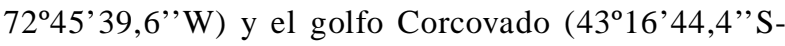
73¹7'02,4”W) (Fig. 1), sur de Chile, durante julio 2013.

En cada estación las muestras de plancton se obtuvieron con una red WP2 de $180 \mu \mathrm{m}$ de abertura de malla, provista de mecanismo de cierre y flujómetro TSK. Se realizaron lances oblicuos, en un estrato sobre la picnoclina (determinada según la lectura en tiempo real) y en otro, abarcando toda la columna de agua, tanto de día como de noche, dependiendo de la planificación general del recorrido del buque.

Las muestras recolectadas fueron inmediatamente fijadas con formalina al $4 \%$ neutralizada con bórax. En el laboratorio, este fijador se reemplazó por etanol $80 \%$ con glicerofosfato de sodio $(\mathrm{pH}>8,5)$.

De cada muestra se separaron manualmente todos los moluscos planctónicos, bajo un microscopio de disección Wild M8. Cada individuo fue asignado primero, a nivel de Clase y posteriormente a niveles taxonómicos inferiores. Para ello se utilizó la clasificación de Bouchet \& Rocroi $(2005,2010)$ para gasterópodos y bivalvos, respectivamente. La identificación de

\footnotetext{
${ }^{1}$ Ojeda M, C Molinet, L Cares \& K Espinoza. 2014. Variabilidad espacial y temporal de larvas de mitílidos en el estuario del Reloncaví, sur de Chile. Libro Resúmenes XXXIV Congreso de Ciencias del Mar, Osorno, 2014, p. 199
} 
Tabla 1. Latitud, longitud, profundidad máxima (en $\mathrm{m}$ ) y área geográfica de las estaciones oceanográficas del Crucero CIM AR 19-Fiordos, desde el fiordo Reloncaví al golfo Corcovado / Latitude, longitude, maximum depth (in $\mathrm{m}$ ) and geographic area of the oceanographic stations of the CIMAR 19-Fiordos Cruise, from the Reloncaví Fjord to the Corcovado Gulf

\begin{tabular}{|c|c|c|c|c|}
\hline $\mathrm{N}^{\circ}$ & $\begin{array}{l}\text { Latitud } \\
\left({ }^{\circ} \mathrm{S}\right)\end{array}$ & Longitud $\left({ }^{\circ} \mathrm{W}\right)$ & $\begin{array}{l}\text { Profundidad } \\
\text { máxima }(\mathrm{m})\end{array}$ & Área \\
\hline 3 & $41^{\circ} 40^{\prime} 44,4^{\prime \prime}$ & $72^{\circ} 45^{\prime} 39,6^{\prime \prime}$ & 240 & Seno Reloncaví \\
\hline 4 & $41^{\circ} 43^{\prime} 00,0^{\prime \prime}$ & $72^{\circ} 37^{\prime} 00^{\prime \prime}$ & 455 & Estero Reloncaví \\
\hline 5 & $41^{\circ} 40^{\prime} 26,4^{\prime \prime}$ & $72^{\circ} 24^{\prime} 46,8^{\prime \prime}$ & 254 & Estero Reloncaví \\
\hline 6 & $41^{\circ} 35^{\prime} 31,2^{\prime \prime}$ & $72^{\circ} 20^{\prime} 27,6^{\prime \prime}$ & 196 & Estero Reloncaví \\
\hline $7 \mathrm{C}$ & $41^{\circ} 33^{\prime} 00,0^{\prime \prime}$ & $72^{\circ} 20^{\prime} 02,4^{\prime \prime}$ & 195 & Estero Reloncaví \\
\hline $7 \mathrm{~B}$ & $41^{\circ} 25^{\prime} 30,0^{\prime \prime}$ & $72^{\circ} 17^{\prime} 36^{\prime \prime}$ & 57 & Estero Reloncaví \\
\hline 8 & $41^{\circ} 45^{\prime} 07,2^{\prime \prime}$ & $72^{\circ} 50^{\prime} 16,8^{\prime \prime}$ & 112 & Seno Reloncaví \\
\hline 9 & $41^{\circ} 52^{\prime} 01,2^{\prime \prime}$ & $72^{\circ} 58^{\prime} 26,4^{\prime \prime}$ & 228 & Golfo Ancud \\
\hline 14 & $41^{\circ} 59^{\prime} 00,0^{\prime \prime}$ & $72^{\circ} 59^{\prime} 30,0^{\prime \prime}$ & 362 & Golfo Ancud \\
\hline 16 & $42^{\circ} 06^{\prime} 57,6^{\prime \prime}$ & $72^{\circ} 59^{\prime} 34,8^{\prime \prime}$ & 253 & Golfo Ancud \\
\hline 20 & $42^{\circ} 20^{\prime} 06,0$ ' & $72^{\circ} 55^{\prime} 51,6^{\prime \prime}$ & 240 & Golfo Ancud \\
\hline 21 & $42^{\circ} 34.48^{\prime} 0^{\prime \prime}$ & $72^{\circ} 54.43^{\prime}$ & 126 & Golfo Ancud \\
\hline 32 & $42^{\circ} 43^{\prime} 37,2^{\prime \prime}$ & $72^{\circ} 53^{\prime} 49,2^{\prime \prime}$ & 120 & Golfo Corcovado \\
\hline 33 & $42^{\circ} 54^{\prime} 10,8^{\prime \prime}$ & $72^{\circ} 52^{\prime} 48,6^{\prime \prime}$ & 142 & Golfo Corcovado \\
\hline 36 & $43^{\circ} 01^{\prime} 45,0^{\prime \prime}$ & $73^{\circ} 00^{\prime} 37,8^{\prime \prime}$ & 204 & Golfo Corcovado \\
\hline 38 & $43^{\circ} 16^{\prime} 44,4^{\prime \prime}$ & $73^{\circ} 17^{\prime} 02,4^{\prime \prime}$ & 179 & Golfo Corcovado \\
\hline
\end{tabular}
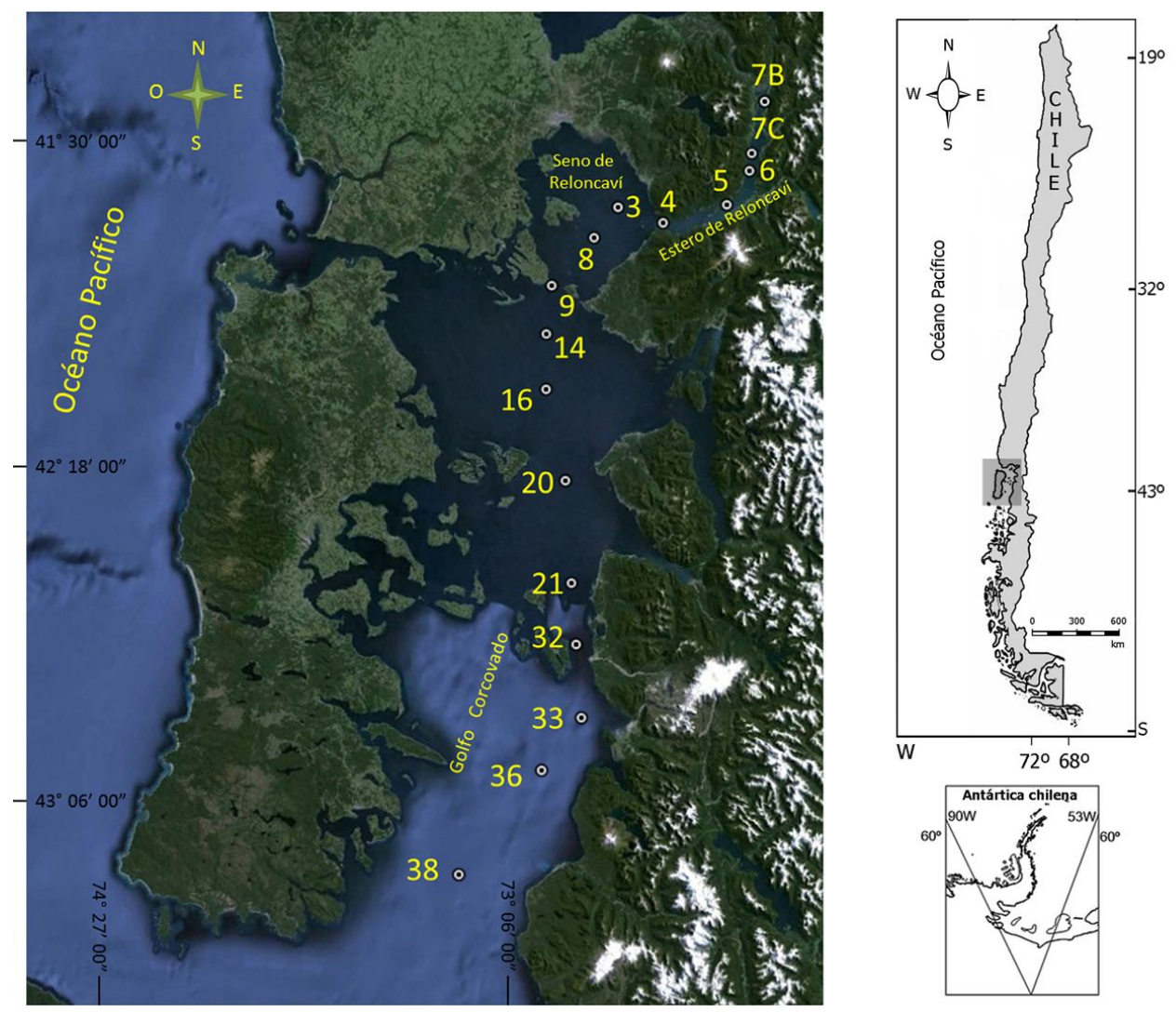

Figura 1. Posición geográfica de las estacionesde muestreo, durante el Crucero CIM AR 19-Fiordos, en julio 2013

/ Geographic location of sampling stations during the CIMAR 19-Fiordos Cruise, in July 2013 
gasterópodos se basó principalmente en los trabajos de Hurst (1967), Fretter \& Pilkington (1970), Van der Spoel (1972), Taylor (1975), Thiriot-Quievreux (1980, 1983), ThiriotQuievreux \& Scheltema (1982), Tardy (1991), Bandel et al. (1997), Van der Spoel et al. (1997), Romero \& Valdebenito (2002) y Oliva-Rivera \& de Jesús-Navarrete (2007). La identificación de bivalvos se basó en los trabajos de Ramorino \& Campos (1983), Campos \& Ramorino (1990), Ugalde (2005), Schejter et al. (2010), Díaz \& Campos (2014) y en la identificación de los cefalópodos se utilizaron los trabajos de Carrasco et al. (2012) y Pardo-Gandarillas et al. (2016).

\section{Análisis de Datos}

La abundancia de los moluscos recolectados en el estrato sobre la capa de mezcla y toda la columna de agua (mayoritariamente capa profunda) se estandarizó en número de larvas $\mathrm{m}^{-3}$ y se determinó su normalidad utilizando la prueba $\mathrm{W}$ de ShapiroWilk (Zar 1984). Cuando los datos no cumplieron con los supuestos de normalidad (W $>0,576 ; P<0,008$ ), los datos pareados de cada estación (sobre la capa de mezcla y toda la columna de agua) fueron comparados con la prueba de Wilcoxon (Zar 1984).

Las figuras de distribución y abundancia se prepararon teniendo como base el mapa de la zona de estudio obtenida en Google Earth $^{2}$ y aplicaciones de Microsoft ${ }^{\circledR}$ Excel y Adobe ${ }^{\circledR}$ Photoshop.

Se dispuso de datos de clorofila, oxígeno disuelto, salinidad y temperatura del agua, obtenidos con un CTD Seabird SBE 19 en cada estación de muestreo, para incluir en análisis multivariados. Se utilizó un Análisis Canónico de Correspondencia (ACC) (Ter Braak 1986) debido a que se basa en análisis de gradientes directos, donde el gradiente en las variables ambientales es conocido a priori y la abundancia de las especies es considerada la respuesta a ese gradiente. Otros análisis multivariados, como los análisis de componentes principales, dendrogramas, o escalamiento no métrico multidimensional se basan en análisis de gradientes indirectos. Previo al análisis, los datos biológicos de abundancia fueron transformados con $\log (x+1)$. Finalmente, se probó si existieron diferencias significativas en la composición y abundancia de la fauna malacológica planctónica entre la capa de mezcla (CM) y la capa profunda (CP), utilizando una prueba de PERMANOVA de una vía. Esta es una prueba no paramétrica de diferencias significativas entre dos o más grupos, basado en una medida de distancia, en este caso se usó la distancia de Bray-Curtis, con 9999 permutaciones (Anderson 2001). Ambos análisis fueron realizados en el programa Past 3.07 (Hammer et al. 2001).

${ }^{2}<$ www.googleearth.com $>$

\section{Resultados}

\section{OCURREnCia}

En todas las estaciones se obtuvieron moluscos planctónicos. Se recolectaron 157.845 individuos, correspondientes a las Clases Bivalvia (39,68\%), Gastropoda $(60,31 \%)$ y Cephalopoda $(0,01 \%)$. De los bivalvos se identificaron larvas de las familias Hiatellidae (Hiatella solida), Mytilidae (Aulacomya atra, Choromytilus chorus y Mytilus chilensis), Pectinidae (Zygochlamys patagonica), Pharidae (Ensis macha), Pholadidae (Nettastomella darwinii), Teredinidae (Bankia martensi) y Xylophagidae (Xylophaga globosa). De los gasterópodos, se identificaron representantes de la superfamilia Doridoidea y de las familias Atlantidae, Caecidae, Limacinidae, Muricidae, Nassariidae, Ranellidae, Trochidae y Turritellidae. De cefalópodos se recolectaron paralarvas de Octopodidae (Robsonella fontaniana).

\section{DisTRIBUCIÓN Y ABUNDANCIA}

En el fiordo Reloncaví, los bivalvos superaron el $80 \%$ del total de los moluscos recolectados (estaciones 3, 5, 6, 7B y 7C). En la desembocadura del estuario, en el seno Reloncaví (estación 4), los bivalvos fueron levemente superiores al 50\% del total de moluscos. Entre el seno Reloncaví y el golfo de Ancud (estaciones 8, 9, 14 y 16), los gasterópodos superaron el 65\% del total de moluscos y desde las islas Desertores hacia el sur en el golfo Corcovado (estaciones 21, 32, 33, 36 y 38), estos superaron el $80 \%$ del total de moluscos planctónicos recolectados (Fig. 2).

Entre los bivalvos, la mayor dominancia numérica (entre 76,2 y $98,8 \%$ ) correspondió a larvas de especies perforadoras $(B$. martensi, X. globosa y $N$. darwinii), superando ampliamente y en todas las estaciones, a las larvas de otras especies de esta Clase (Fig. 3). Larvas de mitílidos también fueron recolectadas en todas las estaciones con un rango de dominancia entre 0,1 y 16,6\%. Las larvas de las restantes especies de bivalvos, como E. macha, H. solida y Z. patagonica, en su conjunto, presentaron un rango de dominancia entre 0,2 y $12,8 \%$ (Fig. $3)$.

La abundancia total de moluscos planctónicos varió entre 18,5 y 124,4 ind. $\mathrm{m}^{-3}$ (Fig. 4). Las larvas de bivalvos variaron entre 1,6 y 89,2 ind. $\mathrm{m}^{-3}$ (Fig. 5), de las cuales las larvas de mitílidos presentaron un rango entre 0,03 y 5,14 ind. $\mathrm{m}^{-3}$ (Fig. 6). La abundancia de gasterópodos planctónicos varió entre 0,75 ind. $\mathrm{m}^{-3}$ y 120,77 ind. $\mathrm{m}^{-3}$ (Fig. 7). Las 13 paralarvas de cefalópodos recolectadas representaron menos del $1 \%$ del total de la abundancia de moluscos capturados y se recolectaron en las estaciones 4, 6, 14, 16, 32 y 36, preferentemente en la capa de mezcla $(\mathrm{CM})$. 


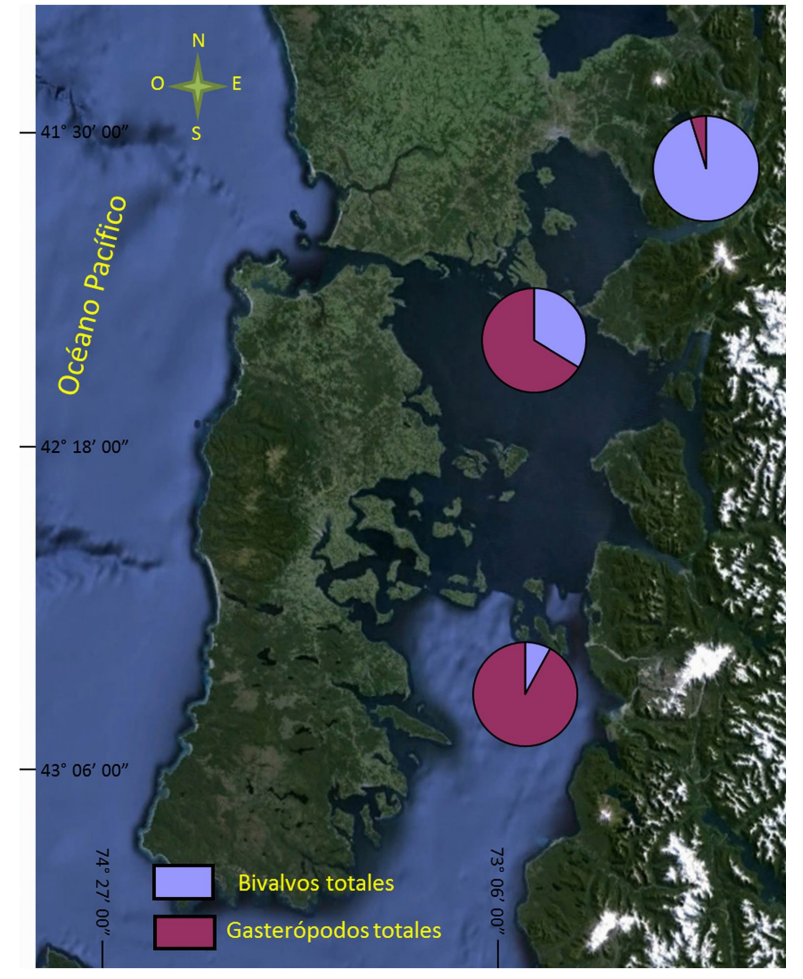

Figura 2. Patrones representativos de dominancia (\%) de moluscos planctónicos recolectados entre el fiordo Reloncaví y el golfo Corcovado, en julio 2013 / Representative dominance patterns (\%) of planktonic mollusks collected from the Reloncaví Fjord to the Corcovado Gulf, in July 2013

No hubo diferencias significativas en la distribución total de los estados planctónicos de moluscos al comparar las abundancias sobre la capa de mezcla (CM) y en toda la columna de agua (principalmente capa profunda, CP) (prueba de Wilcoxon: $Z=0,672 ; P=0,501)$, ni de larvas de bivalvos totales $(\mathrm{Z}=0,051 ; P=0,958)$ así como tampoco de gasterópodos planctónicos totales $(\mathrm{Z}=0,052 ; P=0,937)$.

La Figura 8 muestra la mediana de la abundancia larval de los tres grupos, mostrando que a pesar que hubo mayor cantidad de larvas de bivalvos en la capa superficial, estas diferencias no fueron significativas. El ACC indicó que el principal eje de variabilidad explicó el 99,99\% de la varianza y fue el único eje significativo $(P=0,001)$. La abundancia de los bivalvos estuvo correlacionada con un leve incremento de la temperatura, mientras que los gasterópodos estuvieron correlacionados con un aumento de la salinidad, densidad del agua de mar y concentración de oxígeno (Fig. 9). El PERMANOVA de una vía no detectó diferencias significativas considerando solamente la composición y abundancia logaritmizada del componente malacológico del zooplancton (pseudo- $\mathrm{F}=0,567 ; P=0,588$ ),

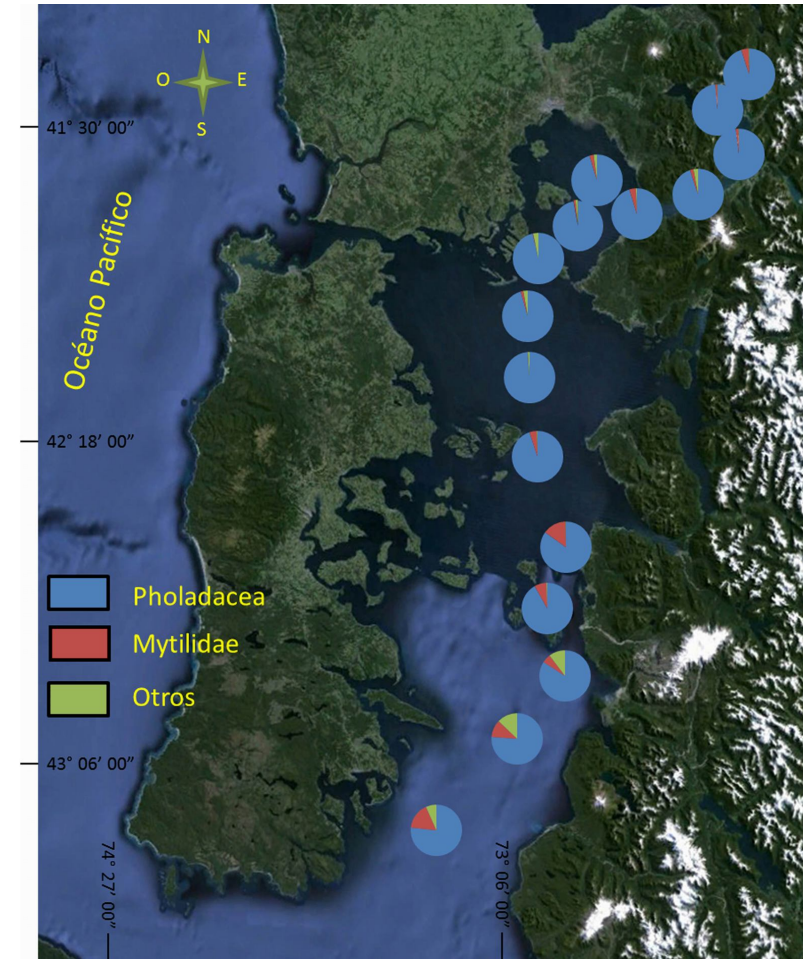

Figura 3. Patrón de dominancia (\%) de larvas de bivalvos recolectadas entre el fiordo Reloncaví y el golfo Corcovado, en julio 2013 / Dominance pattern (\%) of bivalve larvae collected from the Reloncaví Fjord to the Corcovado Gulf, in July 2013

indicando que los moluscos planctónicos fueron similares en la capa de mezcla y en la capa profunda.

\section{Discusión}

La ocurrencia de representantes de las tres Clases principales de moluscos en el plancton de los fiordos del sur de Chile complementa los antecedentes aportados por Ugalde (2005) y Campos \& Díaz (2007), para la zona entre la boca del Guafo y el seno Aysén.

La composición taxonómica de larvas de bivalvos registrada en este estudio se restringe a un número reducido de especies, comparado con la diversidad de bivalvos adultos indicada por Cárdenas et al. (2008) para la zona de estudio. Esta baja diversidad específica de moluscos planctónicos podría estar afectada por la estacionalidad reproductiva que presentan muchas especies bentónicas (Olive 1992) y a los bajos niveles de producción primaria en fiordos durante el invierno (Sánchez et al. 2011, Montero et al. 2011) que pueden reducir la supervivencia larval por menor disponibilidad de alimento. 
Figura 4. Distribución horizontal y abundancia (№ ind. $\mathrm{m}^{-3}$ ) de moluscos planctónicos recolectados entre el fiordo Reloncaví y el golfo Corcovado, en julio 2013 / Horizontal distribution and abundance (№ ind. $\mathrm{m}^{-3}$ ) of planktonic mollusks collected from the Reloncaví Fjord to the Corcovado Gulf, in July 2013
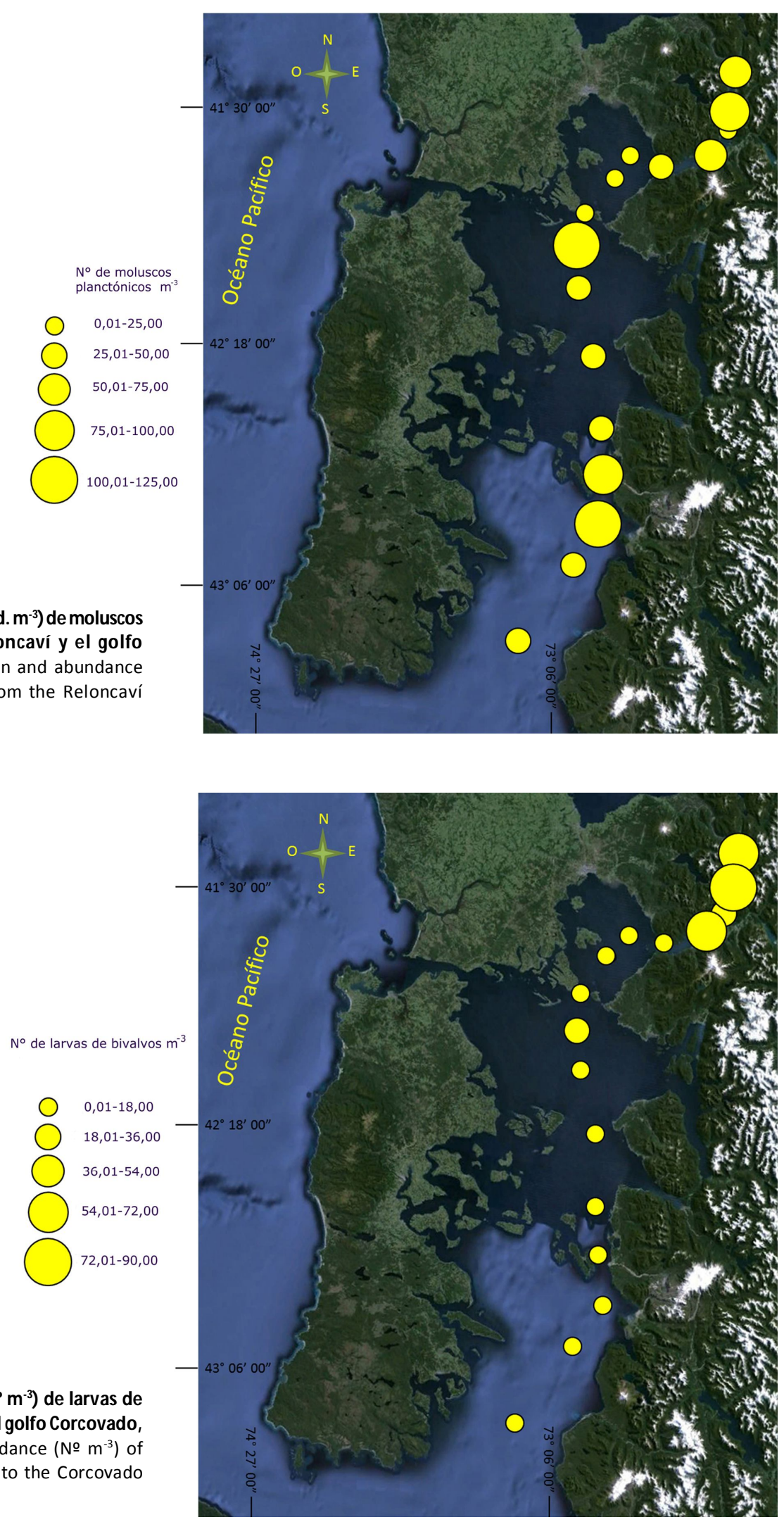

Figura 5. Distribución horizontal y abundancia (№ $\mathrm{m}^{-3}$ ) de larvas de bivalvos recolectadas entre el fiordo Reloncavíy el golfo Corcovado, en julio 2013 / Horizontal distribution and abundance (№ $\mathrm{m}^{-3}$ ) of bivalve larvae collected from the Reloncaví Fjord to the Corcovado Gulf, in July 2013 


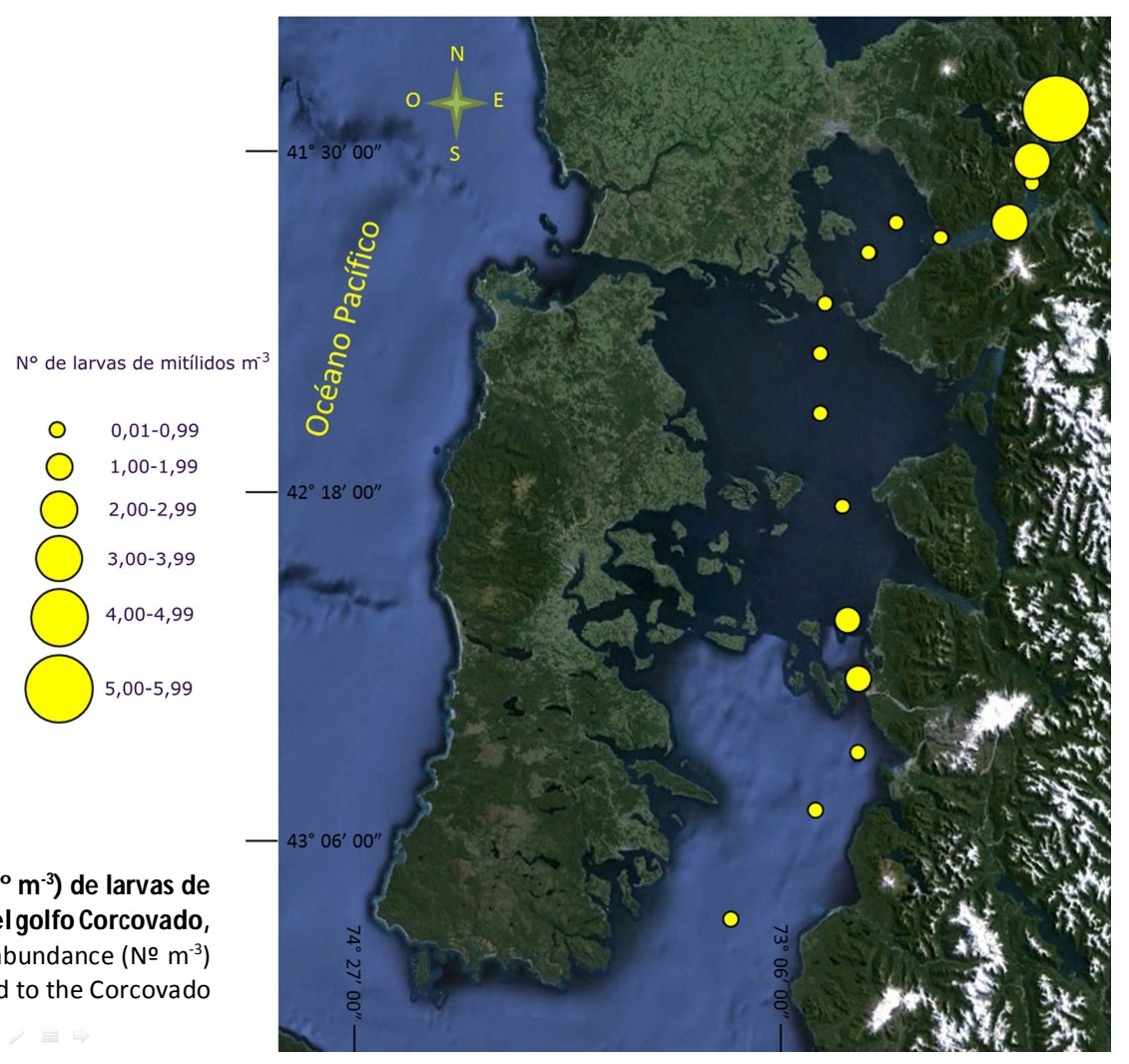

Figura 6. Distribución horizontal y abundancia (№ $\mathrm{m}^{-3}$ ) de larvas de mitílidos recolectadas entre el fiordo Reloncaví y el golfo Corcovado, durante julio 2013 / Horizontal distribution and abundance (№ $\mathrm{m}^{-3}$ ) of mytilid larvae collected from the Reloncaví Fjord to the Corcovado Gulf, in July 2013

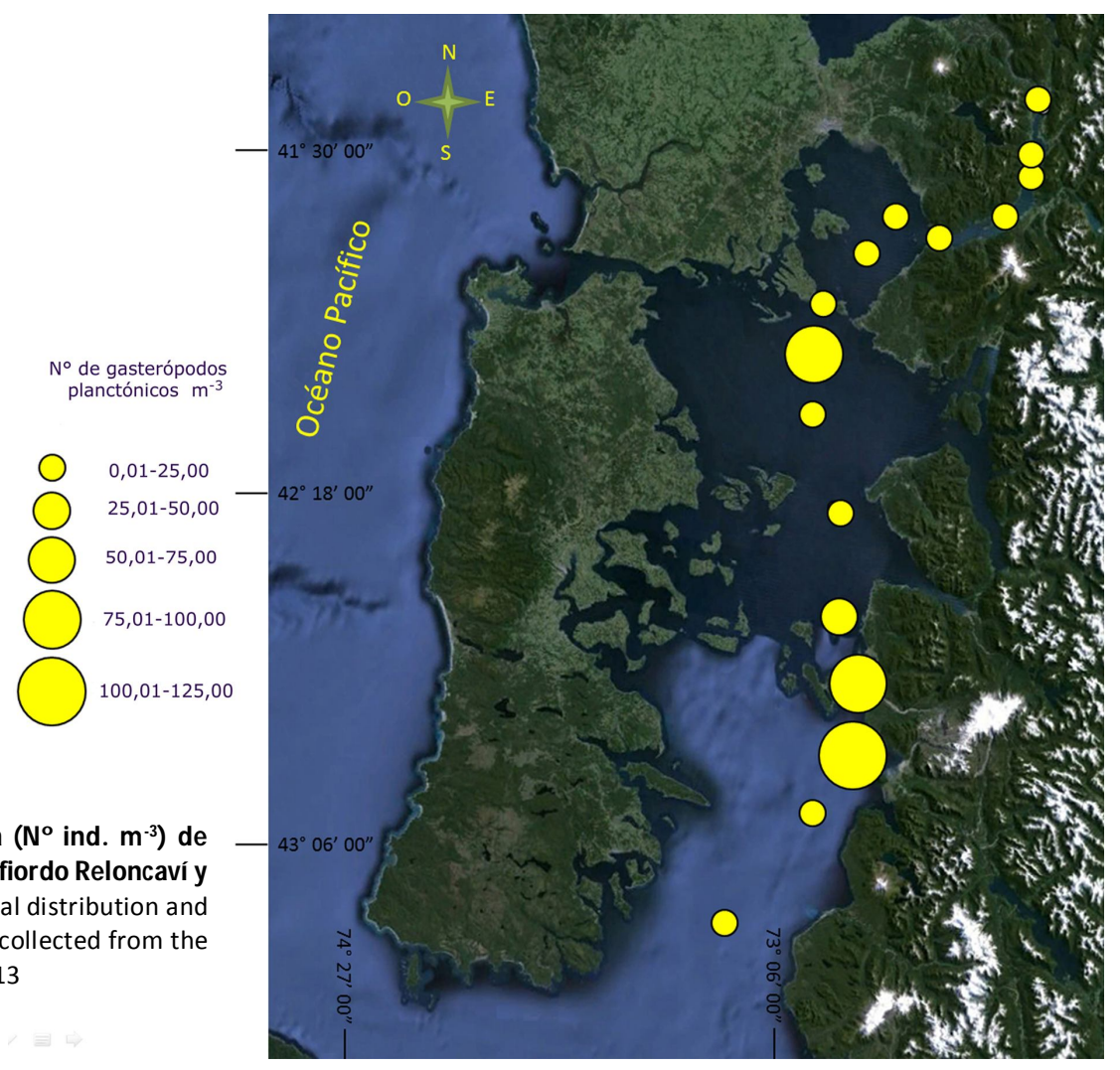

Figura 7. Distribución horizontal y abundancia (№ ind. $\mathrm{m}^{-3}$ ) de gasterópodos planctónicos recolectados entre el fiordo Reloncaví y el golfo Corcovado, durante julio 2013 / Horizontal distribution and abundance ( $N$ o ind. $\mathrm{m}^{-3}$ ) of planktonic gastropods collected from the Reloncaví Fjord to the Corcovado Gulf, in July 2013 

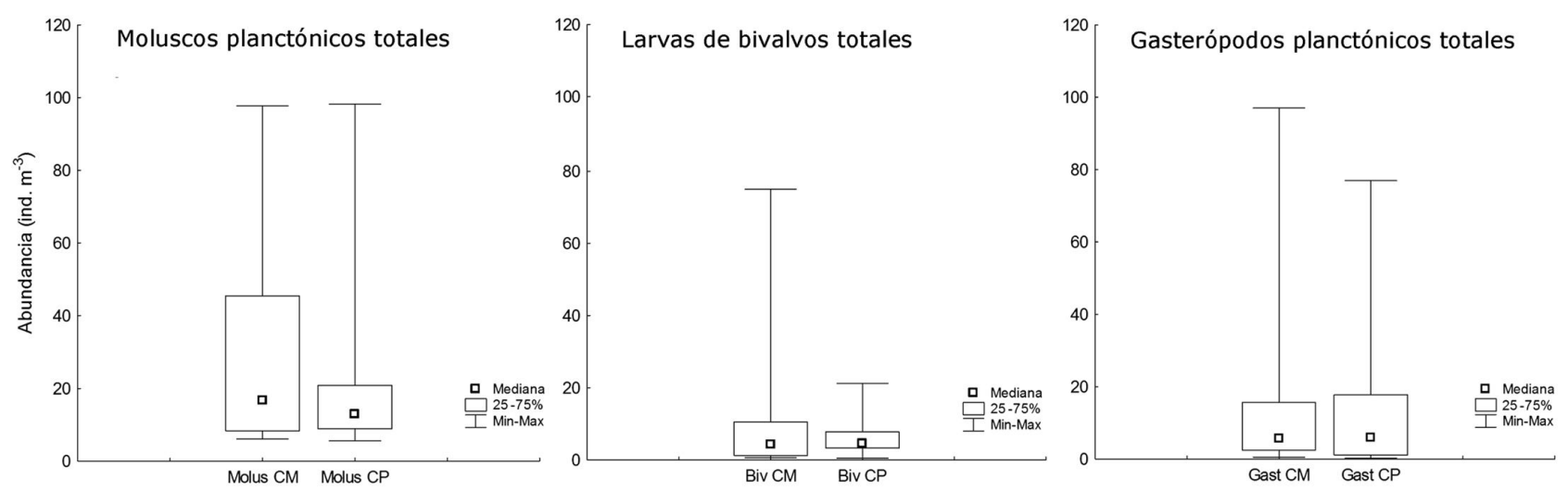

Figura 8. Patrones de distribución vertical de la abundancia de moluscos planctónicos (ind. $\mathrm{m}^{-3}$ ) recolectados entre el fiordo Reloncaví y el golfo Corcovado, durante julio 2013. $\mathrm{CM}=$ capa de mezcla, $\mathrm{CP}=$ capa profunda / Vertical distribution patterns of planktonic mollusks (ind. $\mathrm{m}^{-3}$ ) collected from the Reloncaví Fjord to the Corcovado Gulf, in July 2013. CM= mixing layer, CP= deep layer

Ibrahim (1981), McKoy (1981), Filho et al. (2008), MacIntoch et al. (2012) y Borges (2014) han señalado para bivalvos perforadores de otras latitudes, que hay especies que se reproducen durante todo el año y otras, estacionalmente, en otoño-invierno o primavera-verano, en estrecha relación con la temperatura del agua y con la disponibilidad de alimento.

Debido a la abundancia relativa, por sobre el $70 \%$, de larvas de bivalvos perforadores recolectadas en julio, podría inferirse que provienen de desoves de otoño, ya que se ha indicado que la duración de su período larval sería de 20 días o más (Toth 2015), en las especies con larvas planctotróficas, aunque se desconoce la época reproductiva y la duración larval en el plancton de las especies de Pholadacea que habitan en el mar interior de Chiloé.

La presencia de larvas de mitílidos, con dominancia de Aulacomya atra por sobre Mytilus chilensis y Choromytilus chorus, en período de invierno, difiere de los resultados de Avendaño et al. (2011), quienes recolectaron larvas de estas especies, entre septiembre y marzo, siendo las de M. chilensis las más abundantes. Estudios de ciclo reproductivo de estas tres especies (Lozada et al. 1971, Jaramillo \& Navarro 1995, Oyarzún et al. 2011), en el sur de Chile, señalan que ellas presentarían emisiones gaméticas parciales durante todo el año, por lo tanto, sería esperable encontrar sus larvas en invierno. La ocurrencia de gasterópodos thecosomados del género Limacina también fue registrada por Ugalde (2005), con abundancias mayores en el canal Darwin. En el océano Austral se han identificado Limacina helicina antarctica y Limacina retroversa australis (Hunt et al. 2008), habitando aguas superficiales hasta los $200 \mathrm{~m}$ de profundidad (Bernard 2006). En este trabajo se registró Limacina helicina antarctica pero no $L$. retroversa australis, a pesar que esta última especie sería más dominante que L. helicina antarctica en el Agua Subantártica (Hunt et al. 2008). Esta masa de agua se desplazaría hacia el interior de los fiordos por la Boca del Guafo al golfo Corcovado (Antezana 1999, Sievers \& Silva 2006) y podría explicar la presencia de gasterópodos thecosomados en esta zona. Los registros de gasterópodos thecosomados en los fiordos australes chilenos, realizados por Ugalde (2005) y por esta investigación, al parecer son los primeros en el mar interior de Chiloé, a pesar de la importancia que tienen algunos thecosomados como indicadores de masas de agua, como componentes clave en ciclos biogeoquímicos marinos, como productores y exportadores de carbonato de calcio entre la superficie y el fondo marino (Bednaršek et al. 2012a) y por los efectos que causa en ellos la acidificación del agua al disolver sus delicadas conchas (Bednaršek et al. 2012b). La ocurrencia de paralarvas de cefalópodos de las familias Octopodidae, Loliginidae, Sepiolidae y Onychoteuthidae, había sido registrada solamente en el extremo sur del golfo Corcovado, en la Boca del Guafo, en la desembocadura del seno Aysén, en la conexión entre los canales Moraleda y Darwin y en el interior del canal Darwin (Vega et al. 2000, Ugalde 2005, Carrasco et al. 2012, Pardo-Gandarillas et al. 2016), pero no había sido registrada en el fiordo Reloncaví (estación 6), como ocurrió en este crucero. La presencia de estas paralarvas en el interior del fiordo podría ser considerada ocasional y podría estar limitada por las variaciones en salinidad (por precipitaciones y transporte de agua dulce desde los ríos) existentes en el fiordo Reloncaví ya que los cefalópodos son, en general, estenohalinos (Vecchione 1991, Nabhitabhata et al. 2001)

Aunque en todas las estaciones muestreadas en julio se encontraron moluscos, la abundancia total de ellos fue menor a la reportada por Campos \& Díaz (2007), durante agosto y 


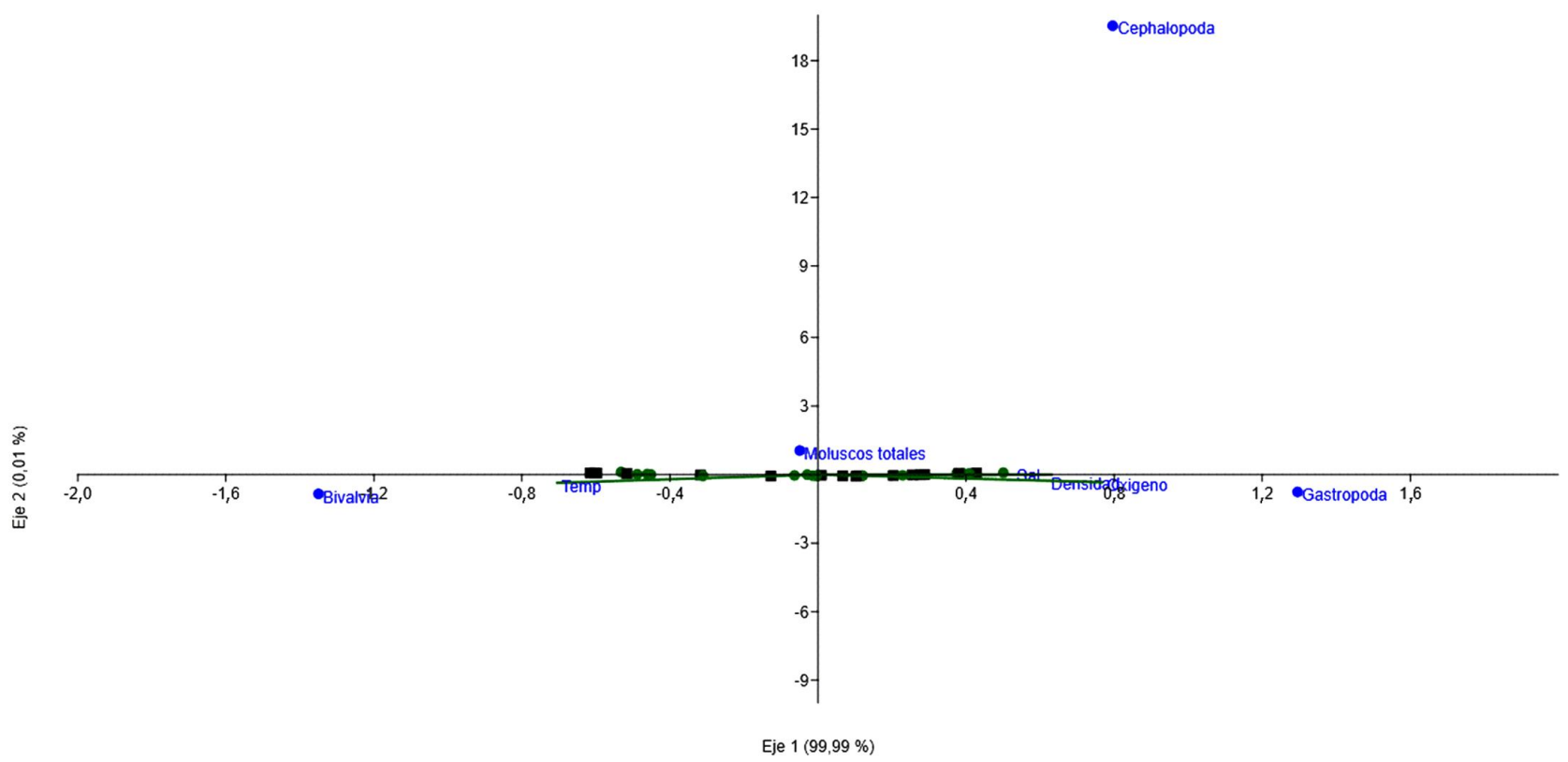

Figura 9. Análisis Canónico de Correspondencia (ACC) de la abundancia transformada a logaritmo de los moluscos planctónicos capturados durante el invierno 2013 y variables oceanográficas asociadas / Canonical Correspondence Analysis (CCA) of logtransformed abundance of planktonic mollusks collected during austral winter 2013 and associated oceanographic variables

noviembre. Esto podría deberse a la disponibilidad de microfitoplancton, principal fuente de alimento para estos organismos planctotróficos (Sánchez et al. 2011).

Avendaño et al. (2011) en el estero Reloncaví registraron densidades máximas de 49.038 larvas $\mathrm{m}^{-3} \pm 11.582$, recolectadas con una red de $53 \mu \mathrm{m}$ de abertura de malla; este valor contrasta con los resultados actuales. Por lo tanto, la selección de la abertura de malla de la red dependerá de los objetivos del muestreo y de la relación costo/tiempo en el análisis de las muestras.

Los tres patrones de dominancia relativa entre bivalvos y gasterópodos planctónicos encontrados podrían estar relacionados con las condiciones oceanográficas, especialmente variaciones en la temperatura en el caso de los bivalvos y salinidad en el caso de los gasterópodos, resultantes de las barreras geográficas y micro cuencas existentes en el mar interior de Chiloé, como la cadena de islas Desertores/Apiao/Quemchi que obstaculiza la entrada de Agua Subantártica hacia la zona norte y detiene al Agua Subsuperficial que fluye hacia el lado norte de esta barrera (Martínez et al. 2015), la desembocadura del fiordo Reloncaví y la constricción que producen las islas Puluqui y Queulín, entre el golfo de Ancud y el seno Reloncaví.

La dominancia de larvas de bivalvos y en particular, de mitílidos, en la cabeza del fiordo Reloncaví, es esperable por la existencia de bancos naturales y centros de cultivo de estos bivalvos a lo largo del fiordo, por el amplio rango de tolerancia que presentan los mitílidos a las variaciones de salinidad propias de un sistema estuarino con una estructura de 3 capas: una superficial de agua dulce que fluye hacia océano, otra intermedia que permite el ingreso de agua más salada y una más profunda de salida del fiordo (Avendaño et al. 2011, Barria et al. 2012, Castillo 2012, Iriarte et al. 2014, Molinet-Flores et al. 2015) regulando así la retención o el retorno de larvas (Boicourt 1982). La ocurrencia y abundancia de larvas de bivalvos perforadores de madera, además de explicarse por mecanismos de retención, por la disponibilidad de maderos sumergidos en los fondos de los fiordos (Orellana 2006, Silva et al. 2011, Vargas et al. 2011, Romano et al. 2013), sin descartarse que algunas larvas se hayan originado en otras áreas ya que algunas larvas de perforadores de madera pueden ser transportadas grandes distancias (Scheltema 1971). La diferencia latitudinal de gasterópodos planctónicos podría relacionarse con la mayor influencia de Agua Subantárctica, con salinidades entre 31 y 33 ups, más tolerables para los gasterópodos, especialmente thecosomados. Las diferencias en la abundancia relativa entre larvas de bivalvos y gasterópodos también han sido señaladas por Meerhoff et al. (2014). Estos autores encontraron en octubre 2012, en los canales Baker y Martínez (Patagonia 
chilena $47,5^{\circ} \mathrm{S}$ ), mayor abundancia de larvas de gasterópodos que de bivalvos, contrario a lo encontrado en febrero 2013, cuando la abundancia de larvas de bivalvos fue muy superior a la de gasterópodos.

El mar interior de Chiloé presenta un diverso ensamble malacológico planctónico. Para comprender mejor su dinámica ecológica se requiere, en primer lugar, disponer de identificaciones confiables de cada especie, además de un programa de muestreo espacio-temporal a escalas suficientemente bajas que permitan detectar los efectos de los principales factores intervinientes en la distribución, dispersión o retención de estos ensambles planctónicos en los fiordos del sur de Chile.

\section{Agradecimientos}

A Franco Salas por la recolección de muestras a bordo. A Marcela Cerón y Felipe Portilla por la separación y cuantificación de los moluscos. Al Dr. Mario Cáceres por facilitar los datos graficados de CTD. A René Astudillo por la confección de gráficos y figuras. Al personal del AGS 'Cabo de Hornos' por su ayuda en los muestreos, al Comité Oceanográfico Nacional por financiar el proyecto CIMAR Fiordos 2013-9 y a dos evaluadores anónimos por sus valiosas sugerencias al trabajo.

\section{LiTERATURA CITADA}

Anderson MJ. 2001. A new method for non-parametric multivariate analysis of variance. Austral Ecology 26: 32-46.

Antezana T. 1999. Plankton of Southern Chilean fjords: trends and linkages. Scientia Marina 63(Supl. 1): 69-80.

Antezana T, M Hamamé, Y Eissler \& S Jara. 1996. Traits of phyto- and zooplankton. In: Arntz W \& M Gorny (eds). Cruise report of the joint Chilean-German-ltalian Magellan 'Victor Hensen' Campaign in 1994 from the Strait of Magellan to Beagle Channel on board 'Victor Hensen' 2, leg 1 and 2, Oct - Nov 1994. Berichte zur Polar-und Meeresforschung 190:23-31.

Antezana T, A Giraldo \& M Hamamé. 1999. Distribución de taxa y fracciones de tamaño del zooplancton y clorofila en primavera en canales magallánicos y fueguinos. En: CONA (ed). Resultados Crucero CIMAR-Fiordo 3, Resúmenes ampliados, pp. 87-94. CONA, Valparaíso.

Avendaño M, M Cantillanez, M Le Pennec, C Varela \& C Garcias. 2011. Distribución temporal de larvas de Mytilus chilensis (Hupé, 1954) (Mollusca: Mytilidae), en el mar interior de Chiloé, sur de Chile. Latin American Journal of Aquatic Research 39(3): 416-426.

Balbontín F, B Campos, A Mujica \& JC Saavedra. 2009. Distribución de larvas de algunos invertebrados y huevos de peces en relación con factores físicos en fiordos patagónicos. Ciencia y Tecnología del Mar 32(1): 49-70.
Bandel K, F Riedel \& H Weikert. 1997. Planktonic gastropod larvae from the Red Sea: a synopsis. Ophelia 47(3): 151-202.

Barria A, P Gebauer \& C Molinet. 2012. Variabilidad espacial y temporal del suministro larval de mitílidos en el Seno de Reloncaví, sur de Chile. Revista de Biología Marina y Oceanografía 47(3): 475-487.

Bednaršek N, GA Tarling, DCE Bakker, S Fielding, EM Jones, HJ Venables, P Ward, A Kuzirian, B Lézé, RA Feely \& EJ Murphy. 2012a. Extensive dissolution of live pteropods in the Southern Ocean. Natural Geosciences 5: 881-885.

Bednaršek N, GA Tarling, S Fielding \& DCE Bakker. 2012b. Population dynamics and biogeochemical significance of Limacina helicina antarctica in the Scotia Sea (Southern Ocean). Deep-Sea Research II 59-60: 105-116.

Bernard KS. 2006. The role of the euthecosome pteropod, Limacina retroversa, in the Polar frontal zone, Southern Ocean. Ph.D. Thesis, Rhodes University, Grahamstown, 196 pp.

Boicourt WC. 1982. Estuarine larval retention mechanisms on two scales. In: Kennedy V (ed). Estuarine comparisons, pp. 445-457, Academic Press, New York.

Borges LMS, LM Merckelbach, Í Sampaio \& SM Cragg. 2014. Diversity, environmental requirements, and biogeography of bivalve wood-borers (Teredinidae) in European coastal waters. Frontiers in Zoology 11:1-13.

Botsford LW, A Hastings \& SD Gaines. 2001. Dependence of sustainability on the configuration of marine reserves and larval dispersal distances. Ecology Letters 4: 144-150.

Bouchet P \& JP Rocroi. 2005. Classification and nomenclator of gastropod families. Malacologia 47(1-2): 1-368.

Bouchet P \& JP Rocroi. 2010. Nomenclator of bivalve families. Malacologia 52(2): 1-172.

Buckland-Nicks J, G Gibson \& R Koss. 2002. Phylum Mollusca: Gastropoda. In: Young C, M Sewell \& M Rice (eds). Atlas of marine invertebrate larvae, pp. 261-287, Academic Press, New York.

Butman CA. 1987. Larval settlement of soft-sediment invertebrates: The spatial scales of patterns explained by active habitat selection and the emerging role of hydrodynamical processes. Oceanography and Marine Biology Annual Reviews 25: 113-165.

Campos B \& P Díaz. 2007. Distribución y abundancia de larvas de moluscos gasterópodos y bivalvos en fiordos y canales del sur de Chile. Ciencia y Tecnología del Mar 30(1): 115-132.

Campos B \& L Ramorino. 1990. Larvas y postlarvas de Pholadacea de Chile (Mollusca: Bivalvia). Revista de Biología Marina 25(1): 15-63.

Cárdenas J, C Aldea \& C Valdovinos. 2008. Chilean marine Mollusca of Northern Patagonia collected during the CIMAR10 Fjords Cruise. Gayana 72(2): 202-240.

Carrasco SA, R Maltrain, F Villenas \& MA Vega. 2012. New records of early life-stages of cephalopods in the Chiloé Interior Sea. Latin American Journal of Aquatic Research 40(1): 229-235.

Castillo M. 2012. Circulación y mezcla en el fiordo Reloncaví, Chile. Tesis Doctoral, Universidad de Concepción, Concepción, 104 pp. 
Díaz P \& B Campos. 2014. Ontogenia de la concha larval y postlarval de cuatro especies de bivalvos de la costa del Pacífico sureste. Revista de Biología Marina y Oceanografía 49(2): 175-191.

Fernández M, A Astorga, SA Navarrete, C Valdovinos \& PA Marquet. 2009. Deconstructing latitudinal species richness patterns in the ocean: does larval development hold the clue? Ecology Letters 12: 601-611.

Filho CS, CH Tagliaro \& CR Beasley. 2008. Seasonal abundance of the shipworm Neoteredo reynei (Bivalvia, Teredinidae) in mangrove driftwood from a northern Brazilian beach. Iheringia, serie Zoologia 98(1): 17-23.

Fretter V \& MC Pilkington. 1970. Prosobranchia. Veliger larvae of Taenioglossa and Stenoglossa. Conseil International pour l'Exploration de la Mer. Zooplankton sheets 129-132: 3-26.

Güller M \& DG Zelaya. 2015. Bivalves from the Chilean Fjords Region: Knowns and unknowns. American Malacological Bulletin 33(2): 1-12.

Hammer $\emptyset$, DAT Harper \& PD Ryan. 2001. PAST: Paleontological statistics software package for education and data analysis. Palaeontologia Electronica 4(1): 1-9. <http:// palaeo-electronica.org/2001_1/past/past.pdf>

Häussermann V \& G Försterra. 2009. Marine benthic fauna of Chilean Patagonia, 1000 pp. Nature in Focus, Santiago de Chile.

Hunt BPV, EA Pakhomov, GW Hosie, V Siegel, P Ward \& K Bernard. 2008. Pteropods in Southern Ocean ecosystems. Progress in Oceanography 78: 193-221.

Hurst A. 1967. The egg masses and veligers of thirty Northeast Pacific Opisthobranchs. The Veliger 9(3): 255-288.

Ibrahim JV. 1981. Season of settlement of a number of shipworms (Mollusca: Bivalvia) in six Australian harbours. Australian Journal of Marine and Freshwater Research 32: 591-604.

Iriarte JL, S Pantoja \& G Daneri. 2014. Oceanographic processes in Chilean fjords of Patagonia: from small to largescale studies. Progress in Oceanography 129: 1-7.

Jaramillo JR \& JM Navarro. 1995. Reproductive cycle of the Chilean ribbed mussel Aulacomya ater (Molina, 1782). Journal of Shellfish Research 14(1): 165-171.

Lozada E, J Rolleri \& R Yañez. 1971. Consideraciones biológicas de Choromytilus chorus en dos sustratos diferentes. Biología Pesquera, Chile 5: 61-108.

Marilao G. 2009. Distribución espacial y temporal de estadios larvales tempranos de mitílidos en bahía Ilque (41³8'20"S; $\left.73^{\circ} 05^{\prime} 00^{\prime \prime} \mathrm{W}\right), \mathrm{X}$ Región, Chile. Tesis de Ingeniería en Acuicultura, Universidad Austral de Chile, Puerto Montt, 62 pp.

Martínez V, C Lara, N Silva, V Gudiño \& V Montecino. 2015. Variability of environmental heterogeneity in northern Patagonia, Chile: effects on the spatial distribution, size structure and abundance of chlorophyll- $a$. Revista de Biología Marina y Oceanografía 50(1): 39-52.

MacIntoch H, R de Nys \& S Whalan. 2012. Shipworms as a model for competition and coexistence in specialized habitats. Marine Ecology Progress Series 461: 95-105.
McEdward 1. 1995. Ecology of marine invertebrate larvae, 465 pp. CRC Press, Boca Raton.

McKoy JL. 1981. Seasonal settlement of shipworms (Bivalvia: Teredinidae) in Tauranga harbour and Wellington harbour. New Zealand Journal of Marine and Freshwater Research 15(2): 171-180

Meerhoff E, FJ Tapia \& LR Castro. 2014. Spatial structure of the meroplankton community along a Patagonian fjord The role of changing freshwater inputs. Progress in Oceanography 129: 125-135.

Molinet C \& C Moreno. 2009. Distribución espacial de larvas veliger de Concholepas concholepas (Bruguiere) (Gastropoda Muricidae) en el mar interior de la Patagonia Noroccidental, Chile. Ciencia y Tecnología del Mar 32(1): 71-82.

Molinet C, A Arevalo, MT González, CA Moreno, J Arata \& E Niklitschek. 2005. Patterns of larval distribution and settlement of Concholepas concholepas (Bruguiere, 1789) (Gastropoda, Muricidae) in fjords and channels of southern Chile. Revista Chilena de Historia Natural 78: 409-423.

Molinet C, A Valle-Levinson, CA Moreno, M Cáceres, M Bello \& M Castillo. 2006. Effects of sill processes on the distribution of epineustonic competent larvae in a stratified system of Southern Chile. Marine Ecology Progress Series 324:95-104.

Molinet C, E Niklitschek, C Moreno \& A Arevalo. 2008. Vertical distribution of early and competent larvae of Concholepas concholepas in two systems of Chilean inland seas. Marine Biology 153: 779-787.

Molinet-Flores C, M Díaz-Gomez, C Arriagada-Muñoz, L Cares-Pérez, S Marín-Arribas, M Astorga-Opazo \& E Niklitschek-Huaquin. 2015. Spatial distribution pattern of Mytilus chilensis beds in the Reloncaví Fjord: hypothesis on associated processes. Revista Chilena de Historia Natural 88: 1-12.

Montero P, G Daneri, HE González, JL Iriarte, FJ Tapia, L Lizárraga, N Sanchez \& O Pizarro. 2011. Seasonal variability of primary production in a fjord ecosystem of the Chilean Patagonia: Implications for the transfer of carbon within pelagic food webs. Continental Shelf Research 31: 202-215.

Nabhitabhata J, P Asawangkune, S Amornjaruchit \& P Promboon. 2001. Tolerance of eggs and hatchlings of neritic cephalopods to salinity changes. Phuket Marine Biological Center Special Publication 25(1): 91-99.

Navarrete SA, JL Largier, G Vera, FJ Tapia, M Parragué, E Ramos, JL Shinen, CA Stuardo \& EA Wieters. 2015. Tumbling under the surf: wave-modulated settlement of intertidal mussels and the continuous settlement-relocation model. Marine Ecology Progress Series 520: 101-121.

Oliva-Rivera JJ \& A de Jesús-Navarrete. 2007. Larvas de moluscos gasterópodos del sur de Quintana Roo, México. Hidrobiológica 17(2): 151-158.

Olive PJW. 1992. The adaptive significance of seasonal reproduction in marine invertebrates: the importance of distinguishing between models. Invertebrate Reproduction \& Development 22(1-3): 165-174. 
Orellana D. 2006. Modelo conceptual de los aportes de materia orgánica en el fiordo Aysén bajo un enfoque ecosistémico. Memoria en Ingeniería en Recursos Naturales Renovables, Universidad de Chile, Santiago, 56 pp.

Osorio C. 1999. Gastrópodos prosobranquios del extremo sur de Chile. Boletín del Museo Nacional de Historia Natural, Chile 48: 37-49.

Osorio C \& L Ramajo. 2007. Contribution to the knowledge of the distribution, reproduction and morphology of Aeneator fontainei D'Orbigny, 1841 and Aeneator (Ellicae) loisae Redher, 1971 (Gastropoda: Buccinidae) in the fjords of the South of Chile. The Veliger 49(2): 70-78.

Osorio C \& DG Reid. 2004. Moluscos marinos intermareales y submareales entre la Boca del Guafo y el estero Elefantes, sur de Chile. Investigaciones Marinas 32(2): 71-89.

Osorio C, ME Ramirez \& M Vega. 2002. Distribución y abundancia de macroorganismos del intermareal de isla Traiguen, Estero Elefantes, Región de Aysén, Chile. Boletín del Museo Nacional de Historia Natural, Chile 51: 175-184.

Osorio C, DG Reid \& L Ramajo. 2005. Moluscos en los canales del sur de Chile entre Boca del Guafo y Estero Elefantes (CIMAR 7 Fiordos). Ciencia y Tecnología del Mar 28(1): 81-88.

Osorio C, R Peña, L Ramajo \& N Gacelón. 2006. Malacofauna bentónica de los canales oceánicos del Sur de Chile $\left(43^{\circ}-45^{\circ} \mathrm{S}\right)$. Ciencia y Tecnología del Mar 29(1): 103-114.

Oyarzún PA, JE Toro, R Jaramillo, R Guiñez, C Briones \& M Astorga. 2011. Ciclo gonadal del chorito Mytilus chilensis (Bivalvia: Mytilidae) en dos localidades del sur de Chile. Latin American Journal of Aquatic Research 39(3): 512-525.

Palma S. 2006. Distribución y abundancia de zooplancton en canales y fiordos australes. En: Silva N \& S Palma (eds). Avances en el conocimiento oceanográfico de las aguas interiores chilenas, Puerto Montt a cabo de Hornos, pp. 107113. Comité Oceanográfico Nacional / Pontificia Universidad Católica de Valparaíso, Valparaíso.

Pardo-Gandarillas MC, CM Ibáñez, JF Ruiz, CA Bustos, FA Peña \& MF Landaeta. 2016. Paralarvae of cephalopods in channels and fjords of the southern tip of Chile (46-53\%S). Fisheries Research 173: 175-182.

Pavés H, HE González, LR Castro \& JL Iriarte. 2014. Carbon flows through the pelagic sub-food web in two basins of the Chilean Patagonian coastal ecosystem: The significance of coastal-ocean connection on ecosystem parameters. Estuaries and Coasts 03/2014 <doi:10.1007/s12237-014-9780-y>

Ramajo L \& C Osorio. 2012. Riqueza y abundancia y patrones espaciales de moluscos desde el Estero de Reloncaví

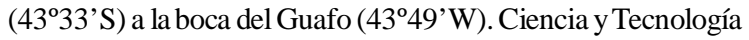
del Mar 33(1): 57-65.

Ramorino L \& B Campos. 1983. Larvas y postlarvas de Mytilidae de Chile. Revista de Biología Marina 19(2): 143-192.

Reid DG \& C Osorio. 2000. The shallow-water marine Mollusca of the Estero Elefantes and Laguna San Rafael, southern Chile. Bulletin of the Natural History Museum of London, Zoology 66(2): 109-146.
Romano C, JR Voight, JB Company, M Plyuscheva \& D Martin. 2013. Submarine canyons as the preferred habitat for wood-boring species of Xylophaga (Mollusca, Bivalvia). Progress in Oceanography 118: 140-158.

Romero MS \& EL Valdebenito. 2002. Larvas veliger de gastrópodos Prosobranchia provenientes de Punta de Lobos, Cuarta Región, Chile. Revista Chilena de Historia Natural 75: 491-514.

Sánchez N, H González \& JL Iriarte. 2011. Trophic interactions of pelagic crustaceans in Comau Fjord (Chile): their role in the food web structure. Journal of Plankton Research 33(8): 1212-1229.

Schejter L, C Bremec, D Waloszek \& M Escolar. 2010. Recently settled stages and larval developmental mode of the bivalves Zygochlamys patagonica and Hiatella meridionalis in the Argentine sea. Journal of Shellfish Research 29(1): 63-67.

Scheltema RS. 1971. Dispersal of phytoplanktotrophic shipworm larvae (Bivalvia: Teredinidae) over long distances by ocean currents. Marine Biology 11(1): 5-11.

Schörld M. 1997. Range extensions of Magellanic Nudibranchs (Opistobranchia) into the Peruvian Faunal province. The Veliger 40(1): 38-42.

Schwabe E, G Försterra, V Häussermann, RR Melzer \& M Schrödl. 2006. Chitons (Mollusca: Polyplacophora) from the southern Chilean Comau Fjord, with reinstatement of Tonicia calbucensis Plate, 1897. Zootaxa 1341: 1-27.

Shanks AL. 2001. An identification guide to the larval marine invertebrates of the Pacific northwest, 314 pp. Oregon State University Press, Corvallis.

Sievers H \& N Silva. 2006. Masas de agua y circulación en los canales y fiordos australes. En: Silva N \& S Palma (eds). Avances en el conocimiento oceanográfico de las aguas interiores chilenas, Puerto Montt a cabo de Hornos, pp. 5358. Comité Oceanográfico Nacional / Pontificia Universidad Católica de Valparaíso, Valparaíso.

Silva N, CA Vargas \& R Prego. 2011. Land-ocean distribution of allochthonous organic matter in surface sediments of the Chiloé and Aysén interior seas (Chilean Northern Patagonia). Continental Shelf Research 31: 330-339.

Solís I, P Sánchez \& S Navarrete. 1976. Identificación y descripción de larvas de moluscos bivalvos en el plancton del estero Castro. Boletín de la Sociedad de Biología de Concepción 50: 183-195.

Suárez-Morales E, R Gasca \& I Castellanos. 2009. Pelagic gastropods. In: Wehrtmann IS \& J Cortés (eds). Marine biodiversity of Costa Rica, Central America, pp. 357-369. Springer Science + Business Media B.V, Netherlands.

Tardy J. 1991. Types of Opisthobranch veligers: Their notum formation and torsion. Journal of Molluscan Studies 57: 103112.

Taylor JB. 1975. Planktonic prosobranch veligers of Kaneoe Bay. Ph.D. Thesis University of Hawaii, Honolulu, 617 pp.

Ter Braak CJF. 1986. Canonical correspondence analysis: a new eigenvector technique for multivariate direct gradient analysis. Ecology 67: 60-71. 
Thiriot-Quievreux C. 1980. Identification of some planktonic prosobranch larvae present off Beaufort, North Carolina. The Veliger 23: 1-9.

Thiriot-Quievreux C. 1983. Summer meroplanktonic prosobranch larvae occurring off Beaufort, Nort Carolina. Estuaries 6: 387-398.

Thiriot-Quievreux C \& RS Scheltema 1982. Planktonic larvae of New England gastropods. V. Bittium alternatum, Thriphora nigrocincta, Cerithiopsis emersoni, Lunatia heros and Crepidula plana. Malacologia 23(1): 37-46.

Thorson GL 1966. Some factors influencing the recruitment and establishment of marine benthic communities. Netherland Journal of Sea Research 3: 267-293.

Toth GB, AI Larsson, PR Jonsson \& C Appelqvist. 2015. Natural populations of shipworm larvae are attracted to wood by waterborne chemical cues. PLoS ONE 10(5): e0124950. <doi:10.1371/journal.pone.0124950>

Trakhtitenbrot A, R Nathan, G Perry \& DM Richardson. 2005. The importance of long-distance dispersal in biodiversity conservation. Diversity Distributions 11: 173-181.

Ugalde K. 2005. Distribución vertical de larvas de moluscos en relación con la circulación estuarina y parámetros oceanográficos, entre el canal Darwin y el seno Aysén, XI Región, Chile. Tesis de Biología Marina, Universidad de Valparaíso, Viña del Mar, 137 pp.

Uribe J, D López \& M González. 1982. Descripción de algunos estados larvarios y postlarvarios de bivalvos chilenos. Noticiario Mensual del Museo de Historia Natural, Chile 26(303-304): 4-10.

Valdovinos C. 1999. Biodiversidad de moluscos chilenos: Base de datos taxonómica y distribución. Gayana 63(2): 111-164.

Valdovinos C \& M Rüth. 2005. Nacellidae limpets of the southern end of South America: Taxonomy and distribution. Revista Chilena de Historia Natural 78: 497-517.
Van der Spoel S. 1972. Pteropoda Thecosomata. Fiches d'identification du zooplancton, $12 \mathrm{pp}$. Conseil International pour l' Exploration de la Mer, Charlottenlund.

Van der Spoel S, L Newman \& KW Estep. 1997. Pelagic molluscs of the world. World Biodiversity Database, CDROM Series. Expert Center for Taxonomic Identification (ETI), Amsterdam.

Vargas C, RA Martínez, V San Martin, M Aguayo, N Silva \& R Torres. 2011. Allochthonous subsidies of organic matter across a lake-river-fjord landscape in the Chilean Patagonia: Implications for marine zooplankton in inner fjord areas. Continental Shelf Research 31: 187-201.

Vecchione M. 1991. Observations on the paralarval ecology of a euryhaline squid, Lolliguncula brevis (Cephalopoda: Loliginidae). Fishery Bulletin 89: 515-521.

Vecchione M \& EK Shea. 2002. Phylum Mollusca: Cephalopoda. In: Young CM (ed). Atlas of marine invertebrate larvae, pp. 327-335. Academic Press, San Diego.

Vega M. 2009. Cefalópodos de aguas chilenas: sistemática y biogeografía, 288 pp. RIL, Santiago de Chile.

Vega M, F Rocha \& C Osorio. 2000. Variaciones espaciales y temporales de paralarvas de cefalópodos en el canal Moraleda $\left(43^{\circ} \mathrm{S}\right)$, sur de Chile. Ciencia y Tecnología del Mar 23:69-82.

Young CM. 2002. A brief history and some fundamentals. In: Young CM (ed). Atlas of marine invertebrate larvae, pp. 119. Academic Press, San Diego.

Zar JH. 1984. Biostatistical analysis, 718 pp. Prentice-Hall, Englewood Cliffs.

Zardus JD \& AL Martel 2002. Phylum Mollusca: Bivalvia. In: Young C, M Sewell \& M Rice (eds). Atlas of marine invertebrate larvae, pp. 289-325, Academic Press, New York. 